\title{
Double-staining procedure for the fluorescent treponemal antibody absorption (FTA-ABS) test
}

\author{
ELIZABETH F. HUNTER, ROGER M. MCKINNEY, SHIRLEY E. MADDISON, \\ AND DONNA D. CRUCE \\ From the Center for Disease Control, Atlanta, Georgia, USA
}

SUMMARY The fluorescent treponemal antibody absorption (FTA-ABS) double-staining procedure was reproducible, comparable to the conventional test, and easy to read. We recommend the use of the FTA-ABS double-staining procedure for microscopes with incident illumination, the $100 \times / 1 \cdot 30$ oil achromatic objective and the $6 \cdot 3 \times$ ocular to obtain optimal fluorescence, and the KP560 as a barrier filter to exclude rhodamine emission when fluorescein fluorescence is read. With this system, errors related to poor focusing or failure to visualise treponemes on all smears should be eliminated.

\section{Introduction}

A double-staining technique has been developed for use in the fluorescent treponemal antibody absorption (FTA-ABS) test (National Communicable Disease Center, 1969). This procedure allows the new improved microscopy equipment to be used, specifically the incident illumination microscope. In the incident illumination system described by Ploem (1967) dichroic mirrors placed in the microscope tube reflect the exciting light through the objective; the objective then serves as the condenser. This system eliminates the necessity for proper alignment and focusing of the conventional darkfield condenser, and the microscope is much easier to use. The equipment offers a number of advantages and is especially useful for quantitative fluorescence measurements. For the present FTAABS test, however, unless the microscope is also equipped for transmitted darkfield illumination (thereby increasing cost), the reader is unable to verify the presence of treponemes on non-reactive slides. It is to solve the latter problem that our attention was directed to the incident illumination microscopes, which have become increasingly popular.

\section{Materials and methods}

Treponema pallidum antigen and sorbent were the reference reagents used for the conventional FTAABS test with transmitted illumination and were

Address for reprints: Dr E. F. Hunter, Center for Disease Control Atlanta, Georgia 30333, USA

Received for publication 29 August 1978 obtained through the Biological Products Division, Center for Disease Control(CDC), Atlanta, Georgia. Sera for preparation of controls and evaluation of the test were characterised samples from syphilitic and non-syphilitic subjects obtained through the Venereal Disease Serum Bank, CDC, or were fresh sera from a public health service outpatient clinic.

The primary antibody was goat anti-Fc fragment of pooled normal human IgG. The secondary antibody was IgG syphilitic serum obtained from sera of patients with clinically documented untreated, latent, or untreated secondary syphilis. The IgG of both sera was obtained by DEAE Sephadex chromatography with $0 \cdot 1 \mathrm{~mol} / 1 \mathrm{NaCl}$ containing $0.05 \mathrm{~mol} / \mathrm{l}$ boric acid buffer adjusted to $\mathrm{pH} 8.0$ with $\mathrm{NaOH}$. The primary and secondary globulins were labelled with either fluorescein isothiocyanate or tetramethyl rhodamine isothiocyanate according to procedures described by McKinney et al. (1976). Additionally, a class-specific fluorescein labelled anti-human IgG reagent was obtained from Burroughs Wellcome Inc. Specificity for IgG was established as described earlier (Reimer et al., 1970; Hunter et al., 1972; Hunter et al., 1976).

DOUBLE-STAINING PROCEDURE

Two methods of double-staining were examined.

\section{Method A}

The procedure is essentially the same as the conventional FTA-ABS test (National Communicable Disease Center, 1969). Absorbed human serum was incubated with $T$. pallidum antigen in the first step. The preparation was washed, and a fluoresceinlabelled anti-human IgG globulin was added to 
demonstrate syphilitic antibody attachment. Slides were rinsed in phosphate-buffered saline. Then, in an additional step, the smears were stained with rhodamine-labelled human anti-T. pallidum globulin. In this step all treponemes are stained; therefore, with incident illumination, reactive and non-reactive treponemes could be visualised without a darkfield condenser.

\section{Method B}

The procedure is the same as that for method $\mathrm{A}$, except that the label of the two globulins was reversed. The primary reagent was rhodaminelabelled anti-human IgG, and fluorescein-labelled anti-T. pallidum globulin was used in the final step.

\section{MICROSCOPE}

The Leitz Ortholux II microscope we used was equipped with the Ploemopak multi-wavelength fluorescence illuminator and a high pressure mercury arc lamp (HBO-200). The basic filter system for fluorescein consisted of the BG-38 red-suppressing filter, the $\mathrm{K} 480$ edge filter, two KP490 exciter filters, the TK510 dichroic beam splitter, and the K515 barrier filter. Additionally, a KP560 filter was inserted on the slider as a barrier filter to exclude rhodamine emission (Haaijman, 1977). The filters for rhodamine excitation included the red-suppressing BG-38, the BG-36 bandabsorption glass filter to absorb the strong mercury line at $578 \mathrm{~nm}$, the KP560 as the exciter filter, the K530 edge filter, the TK580 dichroic beam splitter, and the K590 barrier filter. The optical equipment included $10 \times$ and $6.3 \times$ oculars, a fluorite $(\mathrm{Fl})$ $40 \times / 1 \cdot 30$ oil, and a $100 \times / 1 \cdot 30$ oil achromatic objective.

\section{Results and discussion}

When two fluorescent dyes are present in the same preparation the fluorescence emission must be filtered to allow the observer to visualise both dyes selectively. With the fluorescein and rhodamine dyes excitation of fluorescein will result in some excitation of rhodamine. Without a selective emission filter, staining results may be interpreted incorrectly (Haaijman, 1977). In the figure we show the emission spectra for fluorescein- and rhodamine-labelled conjugates. By adding a KP560 as a barrier filter we are able to observe fluorescein staining, and rhodamine staining is completely excluded.

Traditionally, the FTA-ABS test has been read with a $10 \times$ ocular and a $40 \times$ dry objective. We attempted to read the double-stained slides with a $10 \times$ ocular and a F1 $40 \times / 1.30$ oil objective, but

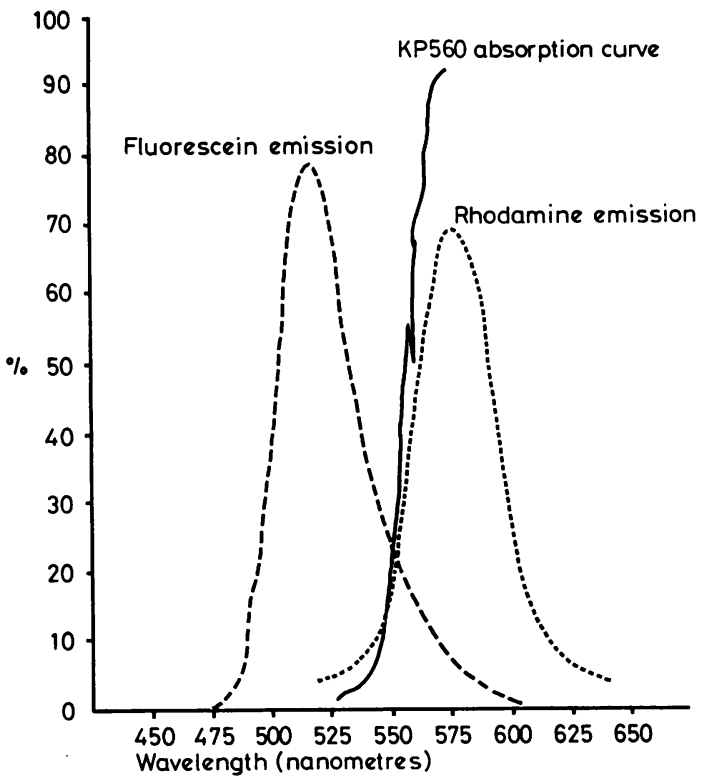

Figure Emission spectra for fluorescein and rhodamine conjugates and KP560 absorption curve

image quality was poor and there was excessive background when non-reactive slides were read with the fluorescein filters (Table 1). The fluorescein filters without the KP560 barrier should pass sufficient rhodamine emission for the $4+$ rhodamine counterstaining on non-reactive treponemes to be visualised as brick-red in colour. This would offer

Table 1 Quality of FTA-ABS test results read by incident illumination on a Leitz Ortholux II microscope equipped with a $10 \times$ ocular and a Fl $40 \times / 1 \cdot 30$ oil objective $(500 \times)$

\begin{tabular}{|c|c|c|c|}
\hline \multirow[b]{2}{*}{ Filter system } & \multicolumn{3}{|c|}{ Microscopical evaluation } \\
\hline & Fluorescence & Image quality & Background \\
\hline 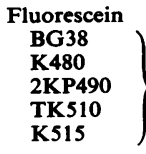 & $\mathbf{S}$ & $\mathbf{U}$ & $\mathbf{U}$ \\
\hline $\begin{array}{c}\text { Above filters } \\
\text { + KP560 }\end{array}$ & $\mathbf{S}$ & $\mathbf{S}$ & $\mathbf{S}$ \\
\hline 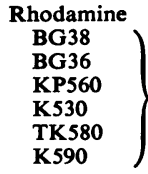 & $\mathbf{S}$ & $\mathbf{S}$ & $\mathbf{S}$ \\
\hline
\end{tabular}

S Satisfactory

U Unsatisfactory 
another possibility for observing treponemes on non-reactive FTA-ABS test smears without the added cost of the multiple Ploem filter system. These optics were considered unsatisfactory for this purpose perhaps because of spherical abberration of the objective and eyepiece magnification (Haaijman, 1977). All other readings were satisfactory.

For the FTA-ABS double-staining procedure a $100 \times / 1 \cdot 30$ oil achromatic objective with a $6.3 \times$ ocular is recommended. We obtained excellent fluorescence, excellent image quality, and an excellent background (Table 2). With this combination non-reactive treponemes could be seen with the single fluorescein filter system because image quality and background were much improved. The brick-red colour was not as easy to read as the maximum red fluorescence obtained with the rhodamine filter system, but the system was satisfactory.

The addition of rhodamine-labelled anti-T. pallidum globulin in the final step of method A stained all treponemes red. We recommend, therefore, that treponemes be located and focused with the rhodamine filter system. The $40 \times$ oil or $40 \times$ dry objective may be easier to use in finding the field. After the field is focused, the $100 \times$ oil objective is moved in place. Then, with the selector knob available on the Ploemopak the fluorescein filters are switched in position, the KP560 is inserted, and fluorescein fluorescence is correctly read. After fluorescence intensity has been recorded, slides can be interchanged with very little focus adjustment.

Table 2 Quality of FTA-ABS test results read by incident illumination on a Leitz Orthulux II microscope equipped with a $6.3 \times$ ocular and a $100 \times / 1.3$ oil achromatic objective $(800 \times)$

\begin{tabular}{|c|c|c|c|}
\hline \multirow[b]{2}{*}{ Filter system } & \multicolumn{3}{|c|}{ Microscopical evaluation } \\
\hline & Fluorescence & Image quality & Background \\
\hline $\begin{array}{l}\text { Fluorescein } \\
\text { BG38 } \\
\text { K480 } \\
\text { 2KP490 } \\
\text { TK510 } \\
\text { K515 }\end{array}$ & $\mathbf{E}$ & $\mathbf{E}$ & $\mathbf{E}$ \\
\hline $\begin{array}{c}\text { Above filters } \\
\text { + KP560 }\end{array}$ & $\mathbf{E}$ & $\mathbf{E}$ & $\mathbf{E}$ \\
\hline $\left.\begin{array}{l}\text { Rhodamine } \\
\text { BG38 } \\
\text { BG36 } \\
\text { KP560 } \\
\text { K530 } \\
\text { TK580 } \\
\text { K590 }\end{array}\right\}$ & $\mathbf{E}$ & $\mathbf{E}$ & $\mathbf{E}$ \\
\hline
\end{tabular}

E Excellent
The slides are much easier to interchange and focus with this equipment than with the darkfield condenser.

We prepared reading controls for the doublestaining procedure and evaluated the test with FTA-ABS test results obtained by reading with transmitted light. We obtained essentially the same results as were obtained in the conventional test on sera from 92 syphilitic subjects (Table 3 ). The differences were between a borderline and a nonreactive and a borderline and a reactive result. Results of tests on sera from 108 non-syphilitic subjects were the same (Table 4).

In the early part of our study we chose method A for several reasons: (1) individuals were trained for reading fluorescein fluorescence; (2) individuals may already have an incident illumination microscope with only the fluorescein filter system; and (3) the Ploemopak was expensive. Now that less expensive microscopes accept up to three filter modules we have to reconsider our choice. We examined method B with a class-specific rhodamine-labelled

Table 3 Comparison of results obtained with the $F T A-A B S$ double-staining procedure and with the conventional test of sera from 92 syphilitic subjects

\begin{tabular}{|c|c|c|c|c|c|c|}
\hline \multirow{3}{*}{$\begin{array}{l}\text { Category of } \\
\text { syphilis }\end{array}$} & \multicolumn{6}{|c|}{$F T A-A B S$ test } \\
\hline & \multicolumn{3}{|c|}{ Double-staining test } & \multicolumn{3}{|c|}{ Conventional test } \\
\hline & $\boldsymbol{R}$ & $\boldsymbol{B}$ & $N$ & $\boldsymbol{R}$ & $\boldsymbol{B}$ & $N$ \\
\hline $\begin{array}{l}\text { Primary } \\
\text { Secondary } \\
\text { Latent } \\
\text { Total }\end{array}$ & $\begin{array}{l}30 \\
26 \\
31 \\
87\end{array}$ & $\begin{array}{l}1 \\
1\end{array}$ & 4 & $\begin{array}{l}30 \\
26 \\
32 \\
88\end{array}$ & 1 & 3 \\
\hline
\end{tabular}

R Reactive

B Borderline

N Non-reactive

Table 4 Comparison of results of the FTA-ABS double-staining procedure and the conventional test with sera from 108 non-syphilitic subjects

\begin{tabular}{|c|c|c|c|c|c|c|}
\hline \multirow{3}{*}{ Category } & \multicolumn{6}{|c|}{ FTA-ABS test } \\
\hline & \multicolumn{3}{|c|}{ Double-staining test } & \multicolumn{3}{|c|}{ Conventional test } \\
\hline & $R$ & $B$ & $N$ & $\boldsymbol{R}$ & $B$ & $N$ \\
\hline \multirow{4}{*}{\multicolumn{2}{|c|}{$\begin{array}{l}\text { Presumed } \\
\text { normals } \\
\text { Diseases } \\
\text { other than } \\
\text { syphilis } \\
\text { Biological } \\
\text { false-positive } \\
\text { Total }\end{array}$}} & & 70 & & & 70 \\
\hline & & & 18 & & & 18 \\
\hline & & & & & & \\
\hline & & & $\begin{array}{r}20 \\
108\end{array}$ & & & $\begin{array}{r}20 \\
108\end{array}$ \\
\hline
\end{tabular}


anti-IgG conjugate and a fluorescein-labelled anti$T$. pallidum globulin. This appears to be a workable system, but further evaluation is needed.

\section{Conclusion}

We recommend use of the double-staining procedure in laboratories where microscopes with incident illumination are available. The FTA-ABS doublestaining procedure described here was highly satisfactory and easy to perform. We do not recommend the use of these microscopes with the single-staining FTA-ABS procedure (National Communicable Disease Center, 1969).

Use of trade names is for identification only and does not constitute endorsement by the Public Health Service or by the US Department of Health, Education, and Welfare.
References

Haaijman, J. J. (1977). Quantitative Immunofluorescence Microscopy, Methods and Applications, pp. 40-43. Publication of the Institute for Experimental Gerontology of the Organization for Health Research TNO, Rijswijk (ZH): The Netherlands.

Hunter, E. F., Maddison, S. E., Larsen, S. A., Felker, M. B., and Feeley, J. C. (1976). Immunoglobulin specificity for the fluorescent treponemal antibody-absorption test conjugate. Journal of Clinical Microbiology, 4, 338-342.

Hunter, E. F., Smith, J. F., Lewis, J. S., McGrew, B. E., and Schmale, J. D. (1972). Immunochemical parameters of some commercial conjugates for the fluorescent treponemal antibody-absorption test. Infection and Immunity, 5, 858-862.

McKinney, R., Thacker, L., and Herbert, G. A. (1976). Conjugation methods in immunofluorescence. Journal of Dental Research (special issue A), 55, A38-A44.

National Communicable Disease Center (1969). Manual of Tests for Syphilis, p. 20. Edited by US Department of Health, Education and Welfare. Public Health Services Publication No. 411. US Government Printing Office: Washington, DC.

Ploem, J. S. (1967). The use of a vertical illuminator with interchangeable dichroic mirrors for fluorescence microscopy with incident light. Zeitschrift fur wissenschaftliche Mikroskopie und mikroskopische Technik, 68, 129-142.

Reimer, C. B. Phillips, D. J., Maddison, S. E., and Shore, S. L. (1970). Comparative evaluation of commercial precipitating antisera against human IgM and IgG. Journal of Laboratory and Clinical Medicine, 76, 949-960. 